\title{
The time-of-flight (ToF) analysis of transmitted electrons at energies of hundred of eV for pure elements
}

Ivo Konvalina ${ }^{1}$, Martin Zouhar $^{1}$, Benjamin Daniel ${ }^{1}$, Aleš Paták ${ }^{1}$, Jakub Piňos ${ }^{1}$, Luděk Frank ${ }^{1}$, Ilona Müllerová $^{2}$ and Eliška Materna Mikmeková ${ }^{2}$

${ }^{1}$ Institute of Scientific Instruments of the CAS, v. v. i., Brno, Jihomoravsky kraj, Czech Republic, ${ }^{2}$ Institute of Scientific Instruments of the Czech Academy of Sciences, Brno, Czech Republic

Both electron microscopy and spectroscopy are powerful tools to gain information about structure and properties of various materials. We have developed a unique ultra-high vacuum (UHV) instrument capable of analyzing samples via transmitted electrons (TEs) in two complementary modes [1]. These two modes are a standard microscopic regime in the range of energies from $5 \mathrm{keV}$ down to few eV and a time-of-flight (ToF) method. Standard ToF spectrometers operate in energy range above $500 \mathrm{eV}$ and detect reflected electrons. Our new ToF spectrometer is able to operate with energies of incident electrons below $300 \mathrm{eV}$. It detects transmitted electrons since the drift tube and detector are conveniently located below the analyzed sample. In order to apply these methods and interpret corresponding results correctly, deep understanding of electron transport phenomena in solids is required.

A single measurement of the TEs detected by the ToF spectrometer produces series of timestamps corresponding to pulse emission, signal detection at multi-channel plate, and pixel-change (over a pixelrange within a selected scanning range). Hyper-spectral data, a time domain spectrum for each pixel within a single scan, are reconstructed from this series. Time domain spectrum, collected over selected pixels (e.g. material regions of highest quality), is converted to the energy domain thus yielding an energy loss spectrum. This energy domain spectrum will provide an input to study of the electron scattering phenomena in pure elements at very low kinetic energies. A resulting energy spectrum for molybdenum, Mo, thin foil for a landing energy equal to $500 \mathrm{eV}$ is shown in figure 1 . The spectrum is complemented by Monte-Carlo (MC) simulations for different thicknesses. The simulations have been performed in program BRUCE [2]. The theoretical spectra have a very narrow elastic/zero-loss peak (ZLP) because they are not affected by finite both energy spread of the primary beam (approximately $0.6 \mathrm{eV}$ in the ToF device) and resolution.

One of the most important parameters governing the electron transport and scattering in materials is the inelastic mean free path (IMFP). The IMFP is defined [3] as the average distance along the trajectories, that particles with a given energy travel, between inelastic collisions in a material of interest. IMFP is widely used for quantification of electron microscopic and spectroscopic data, for example, for interpretation of image contrast, determination of film thicknesses, and chemical composition analysis. The IMFP of solid materials depends on the kinetic energy of electrons. The relationship between the two is often well described by a "universal curve" which is an extrapolation of the experimental data points. Accurate knowledge of IMFP values is crucial to interpreting data acquired via some methods correctly, such as in x-ray photoelectron spectroscopy (XPS) [4].

The IMFP is closely related to inelastic scattering cross-section. Probability of inelastic scattering along a path (segment) of length $d$ is equal to $d / \lambda_{i}$. If the scattering occurs, then the ZLP is decreased, relative 
to the rest of the spectra. The finite energy spread of the primary beam naturally leads us to use area of ZLP, $S_{Z L P}$, instead of a single intensity value (which would correspond to monochromatic primary beam). Hence the ratio $d / \lambda_{i}$ can be expressed in terms of the measured spectra via $Z=\left(S_{\text {total }} / S_{Z L P}\right)$, where $S_{\text {total }}$ denotes area under a portion of (the intensity) spectrum corresponding to a given energy range. Because intensity decay in material is typically an exponential function of thickness, one arrives at $d / \lambda_{i}$ $=\ln (Z)$. This is a redress of the well known log-ratio formula $\lambda_{i}=d / \ln (Z)$ [5]. Such IMFP value is effective in the meaning that it is affected by signal collection aperture size, i.e. acceptance angle of the measuring device.

Another way of obtaining IMFP value is to use the MC simulations in a setup as close as possible to the experimental conditions. Simulate different thicknesses of the material, and compare $\ln (Z)$ of the available spectra, i.e. $\ln \left(Z_{\text {sim }}(d)\right)$ with the experimental ones $\ln \left(Z_{\text {exp }}\right)=\ln \left(Z_{\text {exp }}\left(d_{\text {exp }}\right)\right)$. Calculate the "effective" ratios $\ln \left(Z_{s i m}(d)\right)=d / \lambda_{i}$ from the spectra as described above. The next step is to find "best match" simulated thickness $d_{\text {sim-best }}$ as root of a function $f(d)=d / \lambda_{i, \text { sim }}-d_{\text {exp }} / \lambda_{i, \text { exp }}=\ln \left(Z_{\text {sim }}(d)\right)-\ln \left(Z_{\text {exp }}\right)$ $=\ln \left(Z_{\text {sim }}(d) / Z_{\text {exp }}\right)$. This is obtained using fit of a linear function to the $\ln \left(Z_{\operatorname{sim}}(d) / Z_{\text {exp }}\right)$ data. This "best match" can be used to express the experimental IMFP as follows, $\lambda_{i, \text { exp }}=\lambda_{i \text {, sim }} \mathrm{X}\left(d_{\text {exp }} / d_{\text {sim-best }}\right)$. The value $\lambda_{i \text {, sim }}$ can be found in the database of the MC simulation program. The best match simulated thickness is $d_{\text {sim-best }}=30.2 \AA$. The corresponding $\lambda_{i}=12.8 \AA$ (with the simulation database value $9.668741 \AA$ ). The mismatch of experimental and simulation value can be caused by the following contributions. First, surface of the sample being non-planar (as verified by independent imaging) and the fact that the sample thickness is only estimated (from sputtering conditions and duration) and it may vary across the surface. Second, the "apertures" as set in the MC simulations and as obtained from simulated tracing of electrons in the ToF system with fields corresponding to the experiment do not agree completely since the tracing leads to a bit more complicated aperture which cannot be easily translated to the MC simulation software.

Even though new commercial scanning electron microscopes (SEM) allow measurements at very low beam energies, the 'universal' IMFP parameters, which typically are integrated into some of the commercial software (sometimes hidden for users), can bring undesirable uncertainties in data analysis and interpretation of the results. We aim to extend the presented work [6] and to explore scattering phenomena in pure elements at very low electron energies and to derive the IMFP values experimentally. 


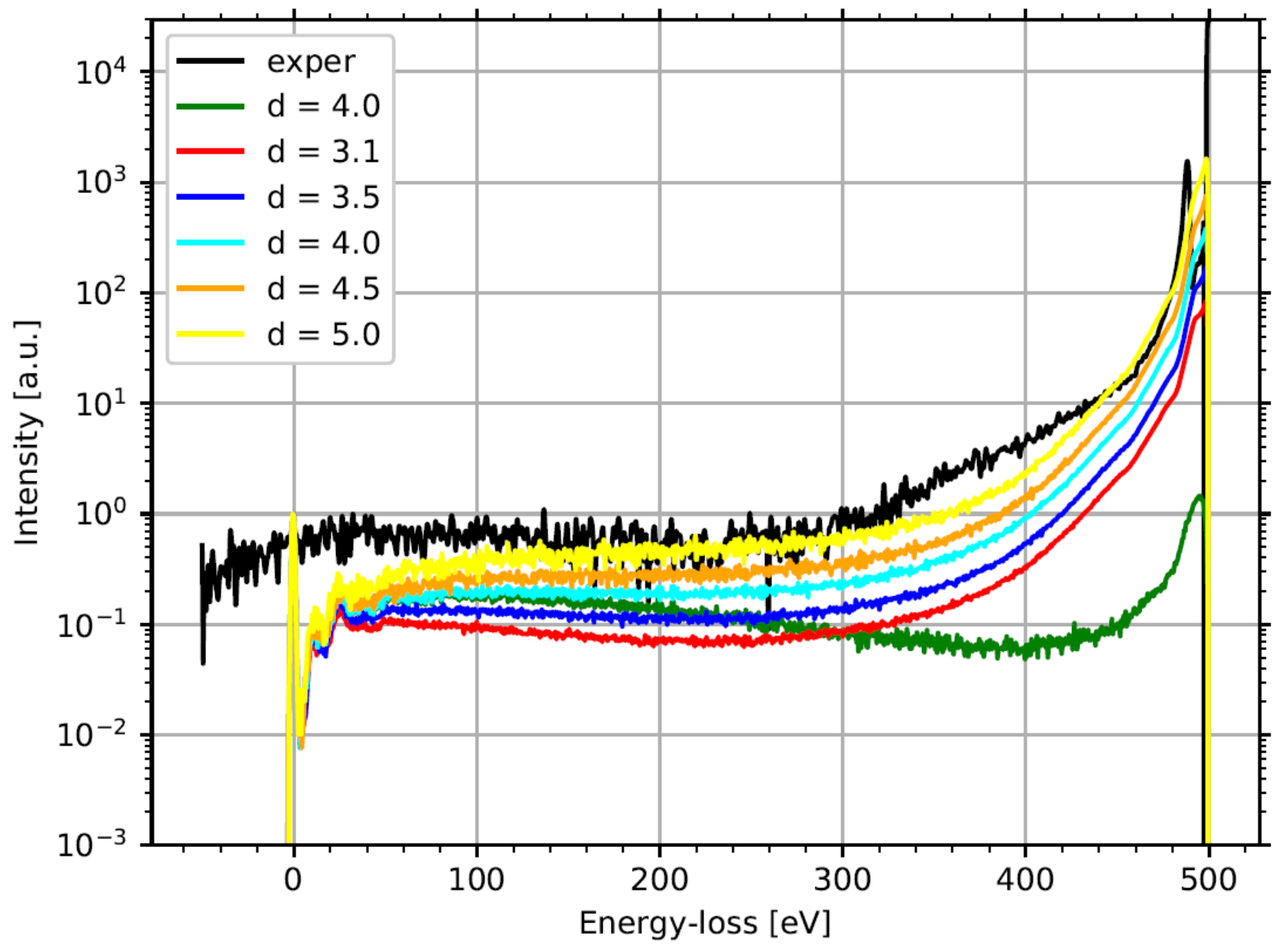

Figure 1. Energy spectra for molybdenum foil, thickness $4 \mathrm{~nm}, \mathrm{EL}=500 \mathrm{eV}$. Experimental (black) and simulated for different thicknesses $d$ [nm]. Displayed spectra normalized to the same height at the zero energy-loss. The 1st $\mathrm{d}=4.0 \mathrm{~nm}$ (green) stands for forwarded electrons only, the rest is the total signal. 


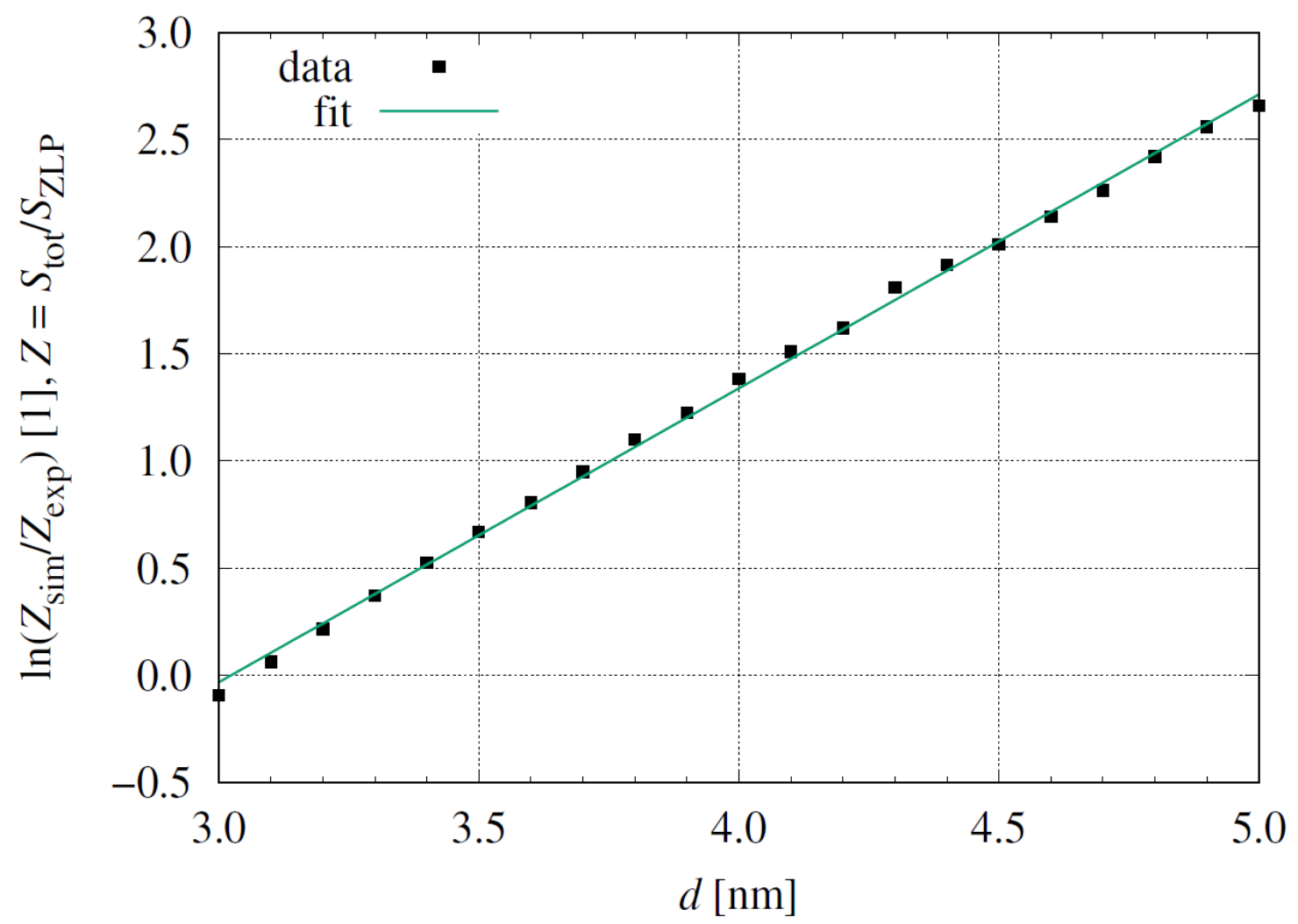

Figure 2. Simulated normalized zero-loss peak cumulative intensity, data points (black squares) and linear fit (green), scaled to the corresponding experimental values of a specific measurement on a reference molybdenum sample.

\section{References}

[1] Konvalina, I., et al. Studying 2D Materials by Means of Microscopy and Spectroscopy with Low Energy Electrons. Microscopy and Microanalysis, 25(S2), (2019), 482-483; doi:10.1017/S1431927619003143

[2] Ridzel, O. Y., Astašauskas, V., Werner, W. S. M. Low energy (1-100 eV) electron inelastic mean free path (IMFP) values determined from analysis of secondary electron yields (SEY) in the incident energy range of $0.1-10 \mathrm{keV}$. J. Electron Spectrosc. Rel. Phen. 241, 146824 (2020); doi:10.1016/j.elspec.2019.02.003

[3] Seah, M. P., Dench, W. A. Quantitative electron spectroscopy of surfaces: A standard data base for electron inelastic mean free paths in solids. Surf. Interface Anal. 1 (1979), 2-11; doi.org/10.1002/sia.740010103

[4] Powell, C. J. Practical guide for inelastic mean free paths, effective attenuation lengths, mean escape depths, and information depths in x-ray photoelectron spectroscopy. J. Vac. Sci. Technol. A38, 023209 (2020); doi: 10.1116/1.5141079

[5] Egerton, R.F. Electron energy-loss spectroscopy in the electron microscope, 2nd ed. (1996), ISBN 978-1-4757-5101-7, Springer Science+Business Media, New York; doi:10.1007/978-1-4757-5099-7

[6] The authors acknowledge funding from the Technology Agency of the Czech Republic (Centre of Electron and Photonic Optics, no. TN01000008). 